KA-TP-30-2012

MPP-2012-114

SFB/CPP-12-50

\title{
Interpretation of precision tests in the Higgs sector in terms of physics beyond the Standard Model
}

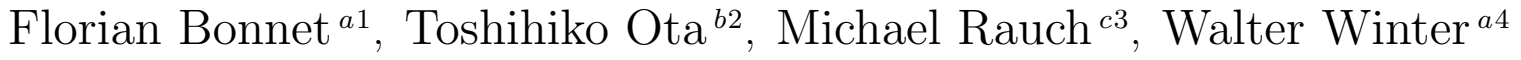 \\ ${ }^{a}$ Institut für Theoretische Physik und Astrophysik, Universität Würzburg, Germany \\ ${ }^{b}$ Max-Planck-Institut für Physik (Werner-Heisenberg-Institut), München, Germany \\ ${ }^{c}$ Institut für Theoretische Physik, Karlsruhe Institute of Technology (KIT), Germany
}

PACS: 12.60.Fr, 14.80.Bn

\begin{abstract}
We demonstrate how the measurements of the Higgs-fermion and Higgs-gauge boson couplings can be interpreted in terms of physics beyond the Standard Model in a model-independent way. That is, we describe deviations from the Standard Model by effective $d=6$ operators made of Higgs fields and gauge fields, under the hypothesis that the new physics may show up in the Higgs sector only and the effective operators are generated at tree level. While the effective operator coefficients are independent in general, the completion of the theory at high energies will lead to specific correlations which will be recovered between Higgs-fermion and Higgs-gauge boson couplings. We demonstrate that the current measurement of these couplings in terms of tree-level new physics requires several new mediators with specific relationships among different couplings. New insights in the effective theory and mediator spaces can be expected for improved measurements from the inclusive $H \rightarrow \tau \tau$ and the exclusive vector boson fusion-dominated $H \rightarrow \gamma \gamma$ search channels, as well as the measurement of the Higgs self-couplings, including higher order couplings which do not exist in the Standard Model.
\end{abstract}

\footnotetext{
${ }^{1}$ bonnet@pd.infn.it

${ }^{2}$ toshi@mppmu.mpg.de

${ }^{3}$ rauch@particle.uni-karlsruhe.de

${ }^{4}$ winter@physik.uni-wuerzburg.de
} 


\section{Introduction}

The last update of the ATLAS and CMS collaborations on the Higgs searches established the existence of a new resonance at $125 \mathrm{GeV}$ with $5 \sigma$ significance for the signal [1,2]. This resonance is compatible with the Higgs boson, that would complete the Standard Model (SM) picture. However, to be sure that this new resonance is the SM Higgs boson, one need, among other things, measure its couplings and compare them to their SM prediction. In case of inconsistencies, one should try to identify the information that can be drawn on new physics from the Higgs data. Several studies have been performed on Monte-Carlo expectations [3 -8 as well as on the first hints from 2011 data $[9 \sqrt{19}]$. In the light of the new Higgs data several new studies have already appeared where general Higgs couplings are probed $[20-26]$. These studies use a model independent approach by adding a small, independent, deviation to each of the Higgs couplings to the SM particles.

However, one needs to interpret these deviations in terms of possible new physics models. With that goal, we study in this paper the implication of a set of effective operators on the Higgs data, see Refs. 27-35] for earlier works, and interpret them with respect to their possible ultraviolet completions. As it was shown in Ref. [35], correlations between the coefficient of the effective operators arise when considering these new physics models, which offer the possibility to test these models via the measurement of the Higgs couplings. The paper is organized as follows. We first review the formalism developed in Ref. [35], showing how the Higgs couplings and observables are modified by the presence of effective operators. We then apply the recent constraints obtained at the LHC to the coefficient of the effective operators and show how they can be used to discriminate between different new physics model. Finally we propose new ways to improve the measurement of the effective coefficients considered in this work.

\section{Modification of the Higgs sector from effective operators}

Effective operators are useful to describe Beyond the SM (BSM) physics in the lowenergy limit in a model-independent way, similar to Fermi's theory for beta decay. The effective Lagrangian can be written as

$$
\mathcal{L}_{\text {eff }}=\mathcal{L}_{\mathrm{SM}}+\sum_{i} \alpha_{i} \mathcal{O}_{i}
$$

where $\alpha_{i} \propto \Lambda^{d-4}$ is suppressed by the scale of new physics $\Lambda$ and the mass dimension $d$ of the operator. While the lowest dimensional operator, the unique $d=5$ Weinberg operator, violates lepton number and leads to Majorana neutrino masses, there is a phletora of $d=6$ operators which can be constructed from SM fields 36] (see also Ref. 37] for the discussion of the number of independent operators). Among these operators, only a few can be built from the Higgs and SM gauge fields only, which can 
be directly related to observables in the Higgs sector. Following Ref. [35], we focus on the ones which can be mediated at tree level (see Refs. [38,39]), assuming that these may be the leading ones in perturbative theories ${ }^{1}$ In the Buchmüller-Wyler basis [36], they read

$$
\begin{array}{rlrl}
\mathcal{O}_{\phi}=-\frac{1}{3}\left(\phi^{\dagger} \phi\right)^{3}, & \mathcal{O}_{\partial \phi} & =\frac{\mathbf{1}}{\mathbf{2}} \partial_{\mu}\left(\phi^{\dagger} \phi\right) \partial^{\mu}\left(\phi^{\dagger} \phi\right), \\
\mathcal{O}_{\phi}^{(1)}=\left(\phi^{\dagger} \phi\right)\left(D_{\mu} \phi\right)^{\dagger}\left(D^{\mu} \phi\right), & \mathcal{O}_{\phi}^{(3)}=\left(\phi^{\dagger} D_{\mu} \phi\right)\left(\left(D^{\mu} \phi\right)^{\dagger} \phi\right),
\end{array}
$$

where $\phi$ is the Higgs doublet. As demonstrated in Ref. [35], $\mathcal{O}_{\phi}$ can only be tested by measuring the Higgs self-couplings [40 47] (or new interactions, such as higher order interactions of the Higgs). Since this may be difficult at the LHC, we disregard it for the moment and comment on it later. In addition, $\mathcal{O}_{\phi}^{(3)}$ is severely constrained by electroweak precision tests (EWPT), in particular, the contribution to the T parameter, which leads to $\alpha_{\phi}^{(3)} v^{2} \lesssim 3 \cdot 10^{-4}$. Therefore, any completion of the theory must not violate this bound, which restricts the possible mediators leading to the effective operators $\mathcal{O}_{\partial \phi}$ and $\mathcal{O}_{\phi}^{(1)}|35|$.

In order to describe the modification of the SM Lagrangian by $\mathcal{O}_{\partial \phi}$ and $\mathcal{O}_{\phi}^{(1)}$, all SM relationships are expressed in terms of the best measured quantities, the Fermi constant $G_{F}$, the fine-structure constant $\alpha$, the mass of the $Z$ boson $M_{Z}$, and the mass of the Higgs boson $M_{H}$, which is accessible at the LHC, and renormalizing the Lagrangian by keeping these fixed to their measured values. This procedure is in detail discussed in Ref. [35], and leads (among other implications) to the following leading order modifications of the SM Higgs-gauge boson and Higgs-fermion couplings:

$$
\begin{aligned}
\lambda_{H V V} & =\lambda_{H V V_{\mathrm{SM}}}\left(1+\alpha_{\phi}^{(1)} \frac{v^{2}}{2}-\alpha_{\partial \phi} \frac{v^{2}}{2}\right), \\
\lambda_{H f f} & =\frac{Y_{f}}{\sqrt{2}}=\frac{Y_{f_{\mathrm{SM}}}}{\sqrt{2}}\left(1-\alpha_{\partial \phi} \frac{v^{2}}{2}\right) .
\end{aligned}
$$

Here $V$ represents the SM gauge bosons $W$ and $Z$. Because of these modifications, the couplings of the Higgs boson to two gluons, mediated by a fermion loop, is also modified and goes like

$$
\lambda_{H g g}=\lambda_{H g_{S M}}\left(1-\alpha_{\partial \phi} \frac{v^{2}}{2}\right),
$$

while the coupling to two photons reads ${ }^{2}$

$$
\lambda_{H \gamma \gamma}=\lambda_{H \gamma \gamma_{\mathrm{SM}}} \frac{\left(1-\alpha_{\partial \phi} \frac{v^{2}}{2}\right) \frac{4}{3} A_{1 / 2}^{H}\left(\tau_{t}\right)+\left(1+\alpha_{\phi}^{(1)} \frac{v^{2}}{2}-\alpha_{\partial \phi} \frac{v^{2}}{2}\right) A_{1}^{H}\left(\tau_{W}\right)}{\frac{4}{3} A_{1 / 2}^{H}\left(\tau_{t}\right)+A_{1}^{H}\left(\tau_{W}\right)}
$$

\footnotetext{
${ }^{1}$ For a discussion how well a different set of the Higgs-gauge interactions can be measured, see Ref. 20.

${ }^{2}$ Here we only consider the tree level correction, and we assume that the loop contribution induced by the same mediators is sub-leading.
} 


\begin{tabular}{|l|l||c|c|c||c|c||c|c|}
\hline \multirow{2}{*}{ Coeff. } & \multicolumn{2}{|c||}{ Participating in } & \multirow{2}{*}{$\mathbf{1}_{0}^{s}$} & $\mathbf{3}_{0}^{s}$ & \multicolumn{3}{c||}{$\mathbf{3}_{1}^{s}$} & \multicolumn{2}{c||}{ Gauge } & \multicolumn{2}{c|}{ Non-gauge } \\
\hline$\alpha_{\phi}^{v}$ & $H W W, H Z Z$ & 0 & $2 \frac{\mu_{\Delta}^{2}}{m_{\Delta}^{4}}$ & $4 \frac{\left.\mu_{\Delta_{1}}\right|^{2}}{m_{\Delta_{1}}^{4}}$ & 0 & $-\frac{g_{U}^{2}}{2 m_{U}^{2}}$ & 0 & $-2 \frac{\tilde{\mathbf{1}}_{0}^{v}}{m_{U}^{2}}$ \\
\hline$\alpha_{\phi}^{(3)}$ & EWPT! & 0 & $-2 \frac{\mu_{\Delta}^{2}}{m_{\Delta}^{2}}$ & $4 \frac{\left|\mu_{\Delta_{1}}\right|^{2}}{m_{\Delta_{1}}^{2}}$ & $-2 \frac{g_{V}^{2}}{m_{V}^{2}}$ & 0 & 0 & $2 \frac{\lambda_{U}^{2}}{m_{U}^{2}}$ \\
\hline$\alpha_{\partial \phi}$ & $H W W, H Z Z$, & $\frac{\mu_{S}^{2}}{m_{S}^{4}}$ & $\frac{\mu_{\Delta}^{2}}{m_{\Delta}^{4}}$ & 0 & $\frac{g_{V}^{2}}{m_{V}^{2}}$ & $\frac{g_{U}^{2}}{4 m_{U}^{2}}$ & $-\frac{\lambda_{V}^{2}}{m_{V}^{2}}$ & $-\frac{\lambda_{U}^{2}}{m_{U}^{2}}$ \\
\hline
\end{tabular}

Table 1: List of mediators and their contributions to the effective operators $\mathcal{O}_{\phi}^{(1)}, \mathcal{O}_{\phi}^{(3)}$, and $\mathcal{O}_{\partial \phi}$, (only single mediator cases). The operator $\mathcal{O}_{\phi}^{(3)}$ is limited by electroweak precision tests (EWPT). Table from Ref. [35].

where $\tau_{i}=\frac{M_{H}^{2}}{4 M_{i}^{2}}, A_{1 / 2}^{H}(\tau)=2[\tau+(\tau-1) f(\tau)] \tau^{-2}, A_{1}^{H}(\tau)=-\left[2 \tau^{2}+3 \tau+3(2 \tau-1) f(\tau)\right] \tau^{-2}$ and

$$
f(\tau)=\left\{\begin{array}{cc}
\arcsin ^{2} \sqrt{\tau} & \tau \leq 1 \\
-\frac{1}{4}\left[\log \left(\frac{1+\sqrt{1-\tau^{-1}}}{1-\sqrt{1-\tau^{-1}}}\right)-i \pi\right] & \tau>1
\end{array} .\right.
$$

For the comparison with the literature, we also use the deviations from the SM couplings $c_{V} \equiv \lambda_{H V V} / \lambda_{H V V_{\mathrm{SM}}}$ and $c_{F} \equiv Y_{f} / Y_{f_{\mathrm{SM}}}$ in the following. As a consequence, the effective operator coefficients can be easily rewritten as

$$
\begin{aligned}
\alpha_{\phi}^{(1)} \frac{v^{2}}{2} & =c_{V}-c_{F}, \\
\alpha_{\partial \phi} \frac{v^{2}}{2} & =1-c_{F}
\end{aligned}
$$

in terms of the deviations of the gauge boson and fermion couplings. Note that in the above formulas we have already assumed that $\alpha_{\phi}^{(3)} \simeq 0$.

\section{Interpretation of deviations from the SM}

The question that naturally arises is the interpretation of the effective operators $\mathcal{O}_{\partial \phi}$ and $\mathcal{O}_{\phi}^{(1)}$ in terms of new physics, and if correlations among these operators can appear in specific theories. Focusing on $\mathrm{SU}(2)$ singlet, doublet, and triplet scalars, and singlet and triplet vectors as the mediation fields of the effective operators, all possibilities for the tree-level mediation of these operators have been identified in Ref. [35]. The procedure can be qualitatively described as follows: all possible topologies and fields insertions, which can lead to the effective operators, have been identified, together with the minimum set of necessary interactions to generate the operators (plus interactions among the mediators). Then the fields have been simultaneously integrated out to derive the operator coefficients and possible effects of new mediator-mediator couplings in the multi-mediator cases. From this minimum set of interactions, no fourfermion vertices can be generated because scalar/vector-fermion couplings have not been introduced. 
In order to simplify the presentation, we have assigned symbols to the mediators and list the SM quantum numbers in brackets in the form $\mathbf{X}_{Y}^{\mathcal{L}}$, where

- $\mathbf{X}$ denotes the $\mathrm{SU}(2)$ nature, i.e., singlet $\mathbf{1}$, doublet $\mathbf{2}$, or triplet $\mathbf{3}$.

- $\mathcal{L}$ refers to the Lorentz nature, i.e., scalar $(s)$ and vector $(v)$.

- $Y$ refers to the hypercharge $Y=Q-I_{3}^{W}$.

The result is summarized in Table 1 for the single mediator cases, i.e., when only one mediator is present at a time. For multiple new mediators, the interactions add trivially. The different couplings in the table refer to couplings between the mediators and the Higgs, and are in detail specified in Ref. [35]. As a peculiarity in the table, the new vector mediators may be gauge fields, or the Lagrangian results from a broken gauge symmetry (non-gauge vectors), where the interactions are introduced in a different way. Furthermore note that the operator $\mathcal{O}_{\phi}$ in Eq. (2) may be mediated by a doublet scalar, which does not appear in Table 1 because it does not lead to any of the shown operators.

From Table 1, one can easily read off that

- The single mediator cases with $\mathbf{3}_{0}^{s}, \mathbf{3}_{1}^{s}, \mathbf{1}_{0}^{v}$, and $\tilde{\mathbf{3}}_{0}^{v}$ are forbidden, since they produce large corrections to the electroweak precision data via $\alpha_{\phi}^{(3)}$.

- If cancellations among the contributions from more than one mediator are allowed, the contribution to $\alpha_{\phi}^{(3)}$ can be eliminated by choosing the couplings accordingly. The most prominent case frequently used in the literature [48 52] and, in fact, the only one we study here, is that of two triplet scalars $\mathbf{3}_{0}^{s}$ and $\mathbf{3}_{1}^{s}$. The cancellation condition $-2 \mu_{\Delta}^{2} / m_{\Delta}^{4}+4\left|\mu_{\Delta_{1}}\right|^{2} / m_{\Delta_{1}}^{4}=0$ implies that $\alpha_{\phi}^{(1)}=4 \mu_{\Delta}^{2} / m_{\Delta}^{4}$ and $\alpha_{\partial \phi}=\mu_{\Delta}^{2} / m_{\Delta}^{4}$.

- For each mediator, particular correlations between the effective operator coefficients $\alpha_{\phi}^{(1)}$ and $\alpha_{\partial \phi}$ are obtained. For example, for $\mathbf{3}_{0}^{v}$, one has $\alpha_{\phi}^{(1)}=-2 \alpha_{\partial \phi}$. These correlations will be directly translated into correlations between $c_{V}$ and $c_{F}$, which are not present if only the effective field theory is considered. In addition, the sign of the deviation from these couplings contains meaningful information.

We show in Fig. 1, left panel, the actual (first) measurement of $c_{V}$ and $c_{F}$ by the CMS collaboration and the translation into the effective operator plane, right panel, using Eq. (9) and Eq. (10) ${ }^{3}$ The different lines (rays) represent the correlations among the effective operator coefficients for specific mediators, where in the case of small couplings ( $\alpha \rightarrow 0$ or, equivalently, $c \rightarrow 1$ ), the SM is recovered. The dashed circle in the right panel shows the perturbative regime, i.e., the region where the effective operator approach is expected to be a good description. Note that the case $\mathbf{1}_{0}^{s}$ corresponds to the minimal composite Higgs model MCHM4 [55,56] in the positive coupling constant region, since they both produce only $\alpha_{\partial \phi}$.

\footnotetext{
${ }^{3}$ For recent combined analyses of the Higgs self-couplings, including the contribution of different channels and constraints from ATLAS, see e.g. Refs. $22,53,54]$.
} 

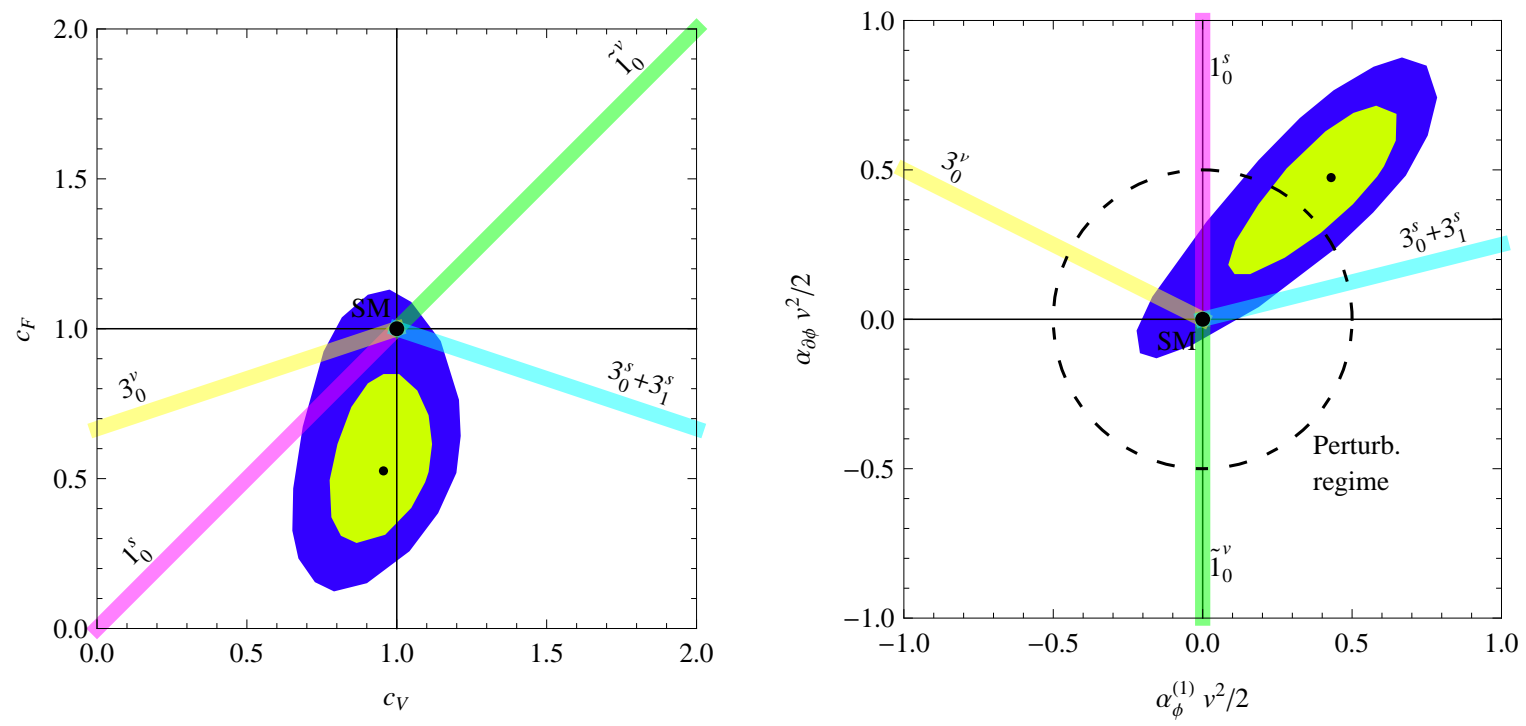

Figure 1: Filled contours: measurement of the Higgs-vector boson and Higgs-fermion couplings in terms of $c_{V}$ and $c_{F}$ (left panel, "experimental plane"), and $\alpha_{\phi}^{(1)}$ and $\alpha_{\partial \phi}$ (right panel, "theory plane"). The data are at the $68 \%$ and $95 \%$ confidence levels, taken from Ref. [2]. The different lines (rays) represent the correlations among the effective operator coefficients for specific mediators, where in the case of small couplings the $\mathrm{SM}$ is recovered. The dashed circle in the right panel shows the perturbative regime (geometric average of couplings smaller than about 0.5), i.e., the region where the effective operator approach is expected to be a good description.

From this figure, one can read off that while the SM is disfavored at the $68 \%$ CL, a simple interpretation with the current data in terms of the simplest possible models seems unlikely. However, using, for instance, three scalars, one can construct more complicated models aligned with the fit contour ( $c f$. , right panel). Such a model would also require specific relationships among the couplings, to give an idea for the required complexity. However, future measurements will improve the precision, and the best-fit shape may change. The picture in Fig. 11 can be used to directly interpret these measurements in terms of theory. The left panel in Fig. 1 can be regarded as "experimental plane", and the right panel as "theory plane". In addition note, as we will show below, that for $m_{H} \simeq 125 \mathrm{GeV}$ the information from the leading channels is highly correlated in the effective operator (theory) plane (cf., Fig. 5 in Ref. [35]). This correlation can be easily seen in the right panel of Fig. 1 .

Note that in the CMS analysis, the parameters $c_{V}$ and $c_{F}$ are independent, and therefore a genuine two parameter fit is performed. In terms of the mediators at tree level, the EWPT constraint turns this fit into a one parameter problem even for two mediators, and the fit of the couplings is reduced to a fit along the lines in Fig. 1 which, however, depends on the mediator. The 95\% confidence level constraints on the individual coupling-mass ratios in Table 1 can be directly read off from Fig. 1 (ranges 
from zero to the point where the lines cross the blue/dark region, to be corrected for one versus two d.o.f. - which leads to a somewhat smaller range than shown in the figure).

\section{Perspectives for different search channels}

The LHC data are collected through different search channels. The ratio of the event rate compared to its SM prediction, $R_{X}^{Y}$, of a channel where the Higgs is produced by the mechanism $X$ and where it decays into a given final state $Y$ is:

$$
R_{X}^{Y}=\frac{\sigma(X \rightarrow H) \times B R(H \rightarrow Y)}{\sigma_{\mathrm{SM}}(X \rightarrow H) \times B R_{\mathrm{SM}}(H \rightarrow Y)} .
$$

Currently the production mechanisms considered are gluon fusion ( $X=\mathrm{gg})$, vector boson fusion $(X=\mathrm{VBF})$ and Higgsstrahlung/associated production $(X=\mathrm{VH})$, while the decays to gauge bosons $(W W, Z Z, \gamma \gamma)$ and fermions $(\tau \tau, b b)$ are measured. In the most recent data, the channels studied are: Inclusive ("incl") channels, which are dominated by the gluon fusion mechanism even for large values of $\alpha_{\partial \phi}$ and $\alpha_{\phi}^{(1)}$. In the numerical analysis below we add the sub-leading contributions from the other production modes blindly, i.e. assuming that acceptance and efficencies are identical for the different production modes. The other possibility are exclusive channels, which only rely on one production mode. For VBF a non-negligible part stems from gluon-fusion production and this is added using the fractions given in the experimental analyses [57, 58]. The selection of the best search channels does not only depend on the Higgs mass, but also on other factors, such as energy resolution and SM backgrounds. The leading search channels right now are the $H \rightarrow \gamma \gamma$ and $H \rightarrow Z Z$ channels, in spite of these branching ratios not being the dominant ones, for which the observables can be approximated (as inclusive channels) by $R_{\text {incl }}^{\gamma \gamma}$ and $R_{\text {incl }}^{Z Z}$.

From Fig. 1, left panel, one can read off that $c_{V}$ is better measured than $c_{F}$ at the moment, which translates through Eq. (4) to the degeneracy $\alpha_{\phi}^{(1)} \propto \alpha_{\partial \phi}$ in the right panel. This means that the individual effective operator coefficients are difficult to extract from this combination. So what observables can be used to break this degeneracy? In order to address this question, we show in Fig. 2 the value of $R_{X}^{Y}$ as a function of the individual effective operator coefficient $\alpha_{\phi}^{(1)} v^{2}$, using a fixed relationship $\alpha_{\partial \phi}=\alpha_{\phi}^{(1)}$ (i.e., along the correlation axis in Fig. 1, right panel). As one can read off from Fig. 2, $R_{\text {incl }}^{\gamma \gamma}$ and $R_{\text {incl }}^{Z Z}$ indeed exhibit very little dependence on the individual

coefficient $\alpha_{\phi}^{(1)}$, which means that a very small error on $R$ is needed to constrain this coefficient. This is illustrated by the gray-shaded area, which shows the effect of a $20 \%$ measurement of $R_{X}^{Y}$ : in this case, the individual coefficient cannot be constrained.

Consider, for instance, $R_{\text {incl }}^{Z Z}$. One can read off from Eq. (4) and Eq. (5) that

$$
R_{\mathrm{gg}}^{Z Z} \propto\left(1-\alpha_{\partial \phi} v^{2}\right)\left(1+\alpha_{\phi}^{(1)} v^{2}-\alpha_{\partial \phi} v^{2}\right) \times \frac{\Gamma_{\text {tot }} \mathrm{MM}}{\Gamma_{\mathrm{tot}}}
$$




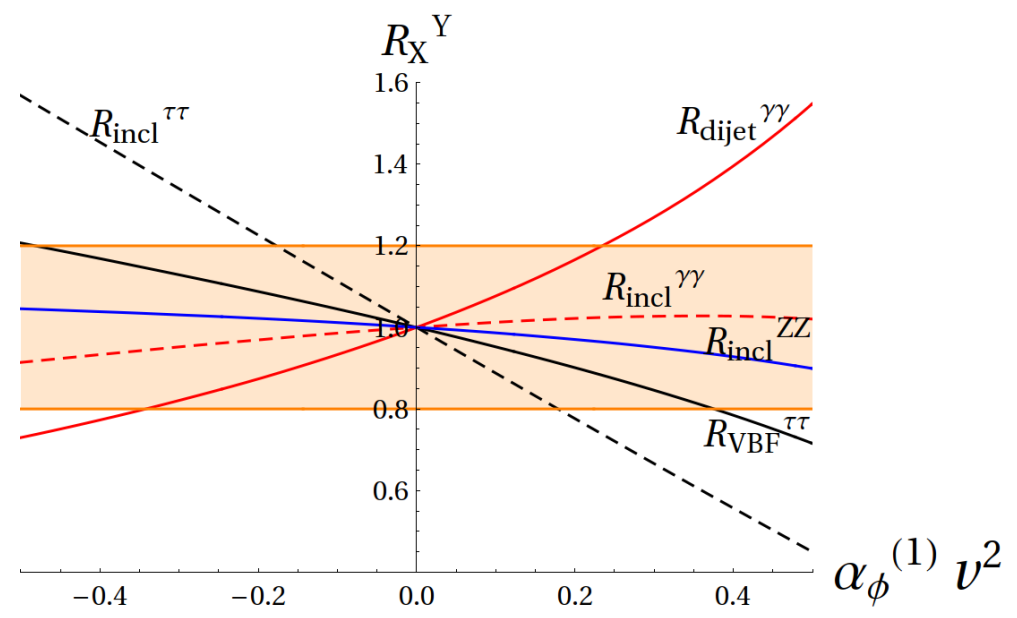

Figure 2: Value of the individual effective operator coefficient $\alpha_{\phi}^{(1)} \frac{v^{2}}{2}$ as a function of $R_{X}^{Y}$ for different search channels for a fixed relationship $\alpha_{\partial \phi}=\alpha_{\phi}^{(1)}$. The orange shading illustrates the effect of a $20 \%$ measurement of $R_{X}^{Y}$.

where the total decay width contains also a dependence in $\alpha_{\partial \phi}$ and $\alpha_{\phi}^{(1)} 4$. This means that it only measures a combination of $\alpha_{\phi}^{(1)}$ and $\alpha_{\partial \phi}$. In this channel the rate change in gluon-fusion production is mostly compensated by a corresponding change in the total width, originating from the dominating fermionic decay modes, while the combination of $\alpha_{\phi}^{(1)}$ and $\alpha_{\partial \phi}$ leaves the decay width unchanged. From Eq. (4) and Eq. (5), it is obvious that $R_{\mathrm{VBF}}^{\tau \tau}$ measures the same combination of parameters, where the role of the new couplings between production and decay is exchanged. Therefore, its role in Fig. 2 as a useful observable is also limited, in addition to the low statistics expected in this exclusive channel.

The most efficient observable is the gluon fusion dominated ratio $R_{\text {incl }}^{\tau \tau}$, since it measures $c_{F}$, and therefore $\alpha_{\partial \phi}$, exclusively; $c f$., Eq. (5). It clearly exhibits the strongest dependence on $\alpha_{\phi}^{(1)}=\alpha_{\partial \phi}$ in Fig. 2. Another option to break the degeneracy is to use a vector boson fusion dominated exclusive $R_{\mathrm{VBF}}^{\gamma \gamma}$ channel: we show $R_{\text {dijet }}^{\gamma \gamma}$ (dominated by $\mathrm{VBF}$ ) in Fig. 2, Here the different dominant Higgs production mode (compared to $R_{\text {incl }}^{\gamma \gamma}$ ) leads to a different dependence on the effective operator coefficients. It is, at the end, a matter of statistics where the best information can be obtained. From the Higgs production cross sections at $125 \mathrm{GeV}$, which is suppressed for vector boson fusion compared to gluon fusion by about one order of magnitude, these options should be comparable in terms of statistics, but it is not clear how this comparison looks like in the presence of selection efficiencies, backgrounds, and energy resolution. In principle, $R_{\text {incl }}^{\tau \tau}$ is the channel from which the strongest constraints are expected, since the dependence in Fig. 2 is stronger, which means that it is worth to optimize it. In terms of theory, both channels will lead to strong constraints of the couplings in Table 1, and may finally rule out the SM prediction.

\footnotetext{
${ }^{4}$ One can make the approximation: $\frac{\Gamma_{\text {tot }}}{\Gamma_{\text {tot }}} \simeq 1+0.25\left(\alpha_{\phi}^{(1)} v^{2}-\alpha_{\partial \phi} v^{2}\right)-0.75 \alpha_{\partial \phi} v^{2}$
} 
Apart from the mediators listed in Table 1, a doublet scalar $\mathbf{2}_{1 / 2}^{s}$ has been found as a possible mediator of $\mathcal{O}_{\phi}$ in Eq. (2) [35], which does not necessarily have to take a vev. The operator $\mathcal{O}_{\phi}$ will lead to shifts of the Higgs self-couplings, which are known to be hard to measure at the LHC, for instance

$$
\lambda_{H H H}=\lambda_{H H H_{\mathrm{SM}}}\left(1-\alpha_{\partial \phi} \frac{v^{2}}{2}+\frac{1}{3} \alpha_{\phi} \frac{v^{2}}{\lambda_{0}}\right),
$$

where $\lambda_{0}=M_{H}^{2} / v^{2}$. If $\alpha_{\partial \phi}$ can be measured from the Higgs-fermion couplings, then $\alpha_{\phi}$ can be in principle extracted from measuring this coupling. However, note that also new higher order effective Higgs interactions are generated by $\mathcal{O}_{\phi}$ :

$$
\delta \mathcal{L}=-\alpha_{\phi} \frac{v}{4} H^{5}-\frac{\alpha_{\phi}}{24} H^{6} .
$$

These are not present in the SM and may be useful in the future to discover new physics and interpret it in terms of BSM theories. However, there will be also an irreducible SM background from the production of several Higgses by SM interactions, and it remains to be seen if useful information can be obtained from these couplings. In addition, the measurement of these couplings requires beyond LHC technology, such as a linear or muon collider.

\section{$5 \quad$ Summary and conclusions}

The discovery of the Higgs at the LHC is the beginning of a new era which can be regarded as the precision physics of the Higgs sector. Especially the new data allow to probe the values of the Higgs-gauge boson and Higgs-fermion couplings. We have focused on the theoretical interpretation of possible deviations from the Standard Model identified there, where we have used a model-independent approach. We have tested the hypothesis that new physics may lead to effective $d=6$ operators made from Higgs and gauge bosons only, and that the new mediators lead to a tree level generation of such an operator. Two of the operators can be directly related to a linear combination of the Higgs-gauge boson and Higgs-fermion couplings, which are perhaps the simplest observables in the Higgs sector.

We have demonstrated how current bounds translate into the measurement of the effective operator coefficients. With the data from the leading channels, an approximate degeneracy between the coefficients has been identified. Future measurements of sub-leading channels, such as the inclusive $H \rightarrow \tau \tau$ channel and the exclusive vector boson fusion-dominated $H \rightarrow \gamma \gamma$ channel, can be used to reduce that and to exclude the SM case. We have also demonstrated how the measurements can be interpreted in terms of the tree level mediators, which lead to particular correlations in the effective operator plane. Although the actual low statistics does not allow one to draw definitive conclusions, we have shown that the LHC is powerful enough to constrain the effective operators that modify the Higgs sector. Current data do not allow for a simple interpretation of the possible deviation from the Standard Model in terms of a single mediator. We have illustrated that a beyond the Standard Model interpretation 
of the current deviation requires several new fields with particular relationships among the couplings, which does not seem appealing.

Finally, additional information on possible new physics in the Higgs sector can be obtained from measuring the Higgs self-couplings, which is known to be challenging. As an alternative, higher order effective Higgs couplings can be used to measure the remaining effective operator, which may be indicative for a new scalar doublet coupling to the Higgs sector only. Although these may be more difficult to measure, this possibility should be studied in the future.

\section{Acknowledgments}

We would like to thank M. B. Gavela for useful and illuminating discussions. FB and WW would like to acknowledge support from DFG grants WI 2639/3-1 and WI 2639/4-1. We would also like to thank the Galileo Galilei Institute for Theoretical Physics for the hospitality during our stay, where a substantial fraction of this work was carried out. MR acknowledges support by the Deutsche Forschungsgemeinschaft via the Sonderforschungsbereich/Transregio SFB/TR-9 "Computational Particle Physics" and the Initiative and Networking Fund of the Helmholtz Association, contract HA101("Physics at the Terascale").

\section{References}

[1] The ATLAS Collaboration, Observation of an Excess of Events in the Search for the Standard Model Higgs boson with the ATLAS detector at the LHC, ATLASCONF-2012-093, 2012.

[2] The CMS Collaboration, Observation of a resonance with a mass near $125 \mathrm{GeV}$ in the search for the Higgs boson in pp collisions at $\mathrm{s}=7 \mathrm{TeV}$ and $8 \mathrm{TeV}$., CMSHIG-12-020, 2012.

[3] D. Zeppenfeld, R. Kinnunen, A. Nikitenko, and E. Richter-Was, Phys.Rev. D62, 013009 (2000), arXiv:hep-ph/0002036.

[4] M. Duhrssen, Prospects for the measurement of Higgs boson coupling parameters in the mass range from 110 - $190 \mathrm{GeV}$, ATL-PHYS-2003-030, 2003.

[5] M. Duhrssen et al., Phys.Rev. D70, 113009 (2004), arXiv:hep-ph/0406323.

[6] R. Lafaye, T. Plehn, M. Rauch, D. Zerwas, and M. Duhrssen, JHEP 0908, 009 (2009), arXiv:0904.3866.

[7] S. Bock et al., Phys.Lett. B694, 44 (2010), arXiv:1007.2645.

[8] M. E. Peskin, (2012), arXiv:1207.2516.

[9] P. P. Giardino, K. Kannike, M. Raidal, and A. Strumia, JHEP 1206, 117 (2012), arXiv:1203.4254. 
[10] D. Carmi, A. Falkowski, E. Kuflik, and T. Volansky, (2012), arXiv:1202.3144.

[11] J. Espinosa, C. Grojean, M. Muhlleitner, and M. Trott, JHEP 1205, 097 (2012), arXiv:1202.3697.

[12] J. Ellis and T. You, JHEP 1206, 140 (2012), arXiv:1204.0464.

[13] A. Azatov, R. Contino, and J. Galloway, JHEP 1204, 127 (2012), arXiv:1202.3415.

[14] A. Azatov et al., JHEP 1206, 134 (2012), arXiv:1204.4817.

[15] M. Farina, C. Grojean, and E. Salvioni, (2012), arXiv:1205.0011.

[16] M. Klute, R. Lafaye, T. Plehn, M. Rauch, and D. Zerwas, (2012), arXiv:1205.2699.

[17] J. R. Espinosa, M. Muhlleitner, C. Grojean, and M. Trott, (2012), arXiv:1205.6790.

[18] D. Carmi, A. Falkowski, E. Kuflik, and T. Volansky, (2012), arXiv:1206.4201.

[19] D. Bertolini and M. McCullough, (2012), arXiv:1207.4209.

[20] T. Corbett, O. Eboli, J. Gonzalez-Fraile, and M. Gonzalez-Garcia, (2012), arXiv:1207.1344.

[21] P. P. Giardino, K. Kannike, M. Raidal, and A. Strumia, (2012), arXiv:1207.1347.

[22] D. Carmi, A. Falkowski, E. Kuflik, T. Volansky, and J. Zupan, (2012), arXiv:1207.1718.

[23] I. Low, J. Lykken, and G. Shaughnessy, (2012), arXiv:1207.1093.

[24] M. Montull and F. Riva, (2012), arXiv:1207.1716.

[25] J. Espinosa, C. Grojean, M. Muhlleitner, and M. Trott, (2012), arXiv:1207.1717.

[26] S. Banerjee, S. Mukhopadhyay, and B. Mukhopadhyaya, (2012), arXiv:1207.3588.

[27] K. Hagiwara, R. Szalapski, and D. Zeppenfeld, Phys. Lett. B318, 155 (1993), arXiv:hep-ph/9308347.

[28] K. Hagiwara, T. Hatsukano, S. Ishihara, and R. Szalapski, Nucl. Phys. B496, 66 (1997), arXiv:hep-ph/9612268.

[29] O. J. P. Eboli, M. C. Gonzalez-Garcia, S. M. Lietti, and S. F. Novaes, Phys. Lett. B434, 340 (1998), arXiv:hep-ph/9802408.

[30] M. C. Gonzalez-Garcia, Int. J. Mod. Phys. A14, 3121 (1999), arXiv:hepph/9902321.

[31] V. Barger, T. Han, P. Langacker, B. McElrath, and P. Zerwas, Phys. Rev. D67, 115001 (2003), arXiv:hep-ph/0301097. 
[32] S. Dutta, K. Hagiwara, and Y. Matsumoto, Phys. Rev. D78, 115016 (2008), arXiv:0808.0477.

[33] S. Kanemura and K. Tsumura, Eur. Phys. J. C63, 11 (2009), arXiv:0810.0433.

[34] F. del Aguila, J. de Blas, and M. Perez-Victoria, JHEP 1009, 033 (2010), arXiv:1005.3998.

[35] F. Bonnet, M. Gavela, T. Ota, and W. Winter, Phys.Rev. D85, 035016 (2012), arXiv:1105.5140.

[36] W. Buchmüller and D. Wyler, Nucl. Phys. B268, 621 (1986).

[37] B. Grzadkowski, M. Iskrzynski, M. Misiak, and J. Rosiek, JHEP 1010, 085 (2010), arXiv:1008.4884.

[38] C. Arzt, Phys. Lett. B342, 189 (1995), arXiv:hep-ph/9304230.

[39] C. Arzt, M. B. Einhorn, and J. Wudka, Nucl. Phys. B433, 41 (1995), arXiv:hep$\mathrm{ph} / 9405214$.

[40] A. Djouadi, W. Kilian, M. Muhlleitner, and P. Zerwas, Eur.Phys.J. C10, 45 (1999), arXiv:hep-ph/9904287.

[41] U. Baur, T. Plehn, and D. L. Rainwater, Phys.Rev.Lett. 89, 151801 (2002), arXiv:hep-ph/0206024.

[42] U. Baur, T. Plehn, and D. L. Rainwater, Phys. Rev. D67, 033003 (2003), arXiv:hep-ph/0211224.

[43] U. Baur, T. Plehn, and D. L. Rainwater, Phys.Rev. D69, 053004 (2004), arXiv:hep-ph/0310056.

[44] M. J. Dolan, C. Englert, and M. Spannowsky, (2012), arXiv:1206.5001.

[45] F. Boudjema and E. Chopin, Z.Phys. C73, 85 (1996), arXiv:hep-ph/9507396.

[46] A. Djouadi, W. Kilian, M. Muhlleitner, and P. Zerwas, Eur.Phys.J. C10, 27 (1999), arXiv:hep-ph/9903229.

[47] U. Baur, Phys. Rev. D80, 013012 (2009), arXiv:0906.0028.

[48] H. Georgi and M. Machacek, Nucl.Phys. B262, 463 (1985).

[49] M. S. Chanowitz and M. Golden, Phys.Lett. B165, 105 (1985).

[50] J. Gunion, R. Vega, and J. Wudka, Phys.Rev. D42, 1673 (1990).

[51] J. Gunion, R. Vega, and J. Wudka, Phys.Rev. D43, 2322 (1991).

[52] H. E. Logan and M.-A. Roy, Phys.Rev. D82, 115011 (2010), arXiv:1008.4869.

[53] J. Ellis and T. You, (2012), arXiv:1207.1693. 
[54] M. Gillioz, R. Grober, C. Grojean, M. Muhlleitner, and E. Salvioni, (2012), arXiv:1206.7120.

[55] K. Agashe, R. Contino, and A. Pomarol, Nucl.Phys. B719, 165 (2005), arXiv:hep$\mathrm{ph} / 0412089$.

[56] J. Espinosa, C. Grojean, and M. Muhlleitner, JHEP 1005, 065 (2010), arXiv:1003.3251.

[57] The CMS Collaboration, Evidence for a new state decaying into two photons in the search for the standard model Higgs boson in pp collisions, CMS-HIG-12-015, 2012.

[58] The CMS Collaboration, Search for a standard model Higgs bosons decaying to tau pairs in pp collisions, CMS-HIG-12-018, 2012. 